\title{
Combined Exciton-Cyclotron Resonance in Quantum Well Structures
}

\author{
D. R. Yakovlev, ${ }^{1,2}$ V.P. Kochereshko, ${ }^{2}$ R. A. Suris,${ }^{2}$ H. Schenk,${ }^{1}$ W. Ossau,${ }^{1}$ A. Waag, ${ }^{1}$ G. Landwehr, ${ }^{1}$ \\ P. C. M. Christianen, ${ }^{3}$ and J. C. Maan ${ }^{3}$ \\ ${ }^{1}$ Physikalisches Institut der Universität Würzburg, 97074 Würzburg, Germany \\ ${ }^{2}$ A. F. Ioffe Physico-Technical Institute, Russian Academy of Sciences, 194021 St. Petersburg, Russia \\ ${ }^{3}$ High Field Magnet Laboratory, RIM, University of Nijmegen, 6525 Nijmegen, The Netherlands
}

(Received 1 May 1997)

\begin{abstract}
A combined exciton-cyclotron resonance is found in photoluminescence excitation and reflectivity spectra of semiconductor quantum wells containing an electron gas of low density. In external magnetic fields, an incident photon creates an exciton in the ground state and simultaneously excites one of the resident electrons from the lowest to one of the upper Landau levels. A theoretical model is developed, which gives a good quantitative description of the energy position and the intensity of the combined exciton-cyclotron resonance. [S0031-9007(97)04535-3]

PACS numbers: 71.35.Gg, 76.40.+b, 78.66.Hf
\end{abstract}

The energy spectrum of quasi-two-dimensional excitons in quantum well semiconductor heterostructures has been extensively studied and is understood fairly well for two extreme cases: (i) When the exciton states are unperturbed, in the absence of free carriers in the quantum wells (QW's), and (ii) when the exciton states are completely screened, at high concentrations of free carriers, such as in modulation-doped QW structures or under high-density excitation [1]. However, the transition regime between these two limiting cases, when a two-dimensional electron gas (2DEG) of low density $n_{e}$ is present, has not been investigated in detail. First indications that interesting phenomena appear in this regime are the observation of negatively charged excitons $\left(X^{-}\right)$, i.e., two electrons bound to one hole, reported for CdTe/CdZnTe and GaAs/AlGaAs modulation-doped QW's [2,3], and enhanced energy and phase relaxation rates for excitons in the presence of background electrons [4-6]. These examples are by no means the only possible manifestations of the exciton-electron interaction. In this Letter, we demonstrate the existence of a new three particle optical resonance, which appears in the absorption spectrum of a QW containing a low density $2 \mathrm{DEG}$ in an external magnetic field. In this case, an incident photon creates not only an exciton, but, in addition, excites a background electron from one Landau level to another, which can be described as a combined excitoncyclotron resonance (combined ExCR).

To study the above mentioned effects of the excitonelectron interaction, we have chosen a $\mathrm{CdTe} / \mathrm{CdMgTe}$ quantum well system, which is known to exhibit strong excitonic effects, as witnessed by the first observation of $X^{-}$in a similar system [2]. The necessary variation of the electron density was achieved by external optical illumination exploiting a specially designed layer composition. The type I CdTe/ $\mathrm{Cd}_{0.74} \mathrm{Mg}_{0.26} \mathrm{Te}$ heterostructure grown by MBE consists of a $75 \AA \mathrm{QW}$, which is sandwiched between $1000 \AA$ thick superlattices (SL's) $(30 \AA / 30 \AA)$. The QW is separated from the SL's by $200 \AA$ barriers (see inset of Fig. 1), which leads to a much higher proba- bility for electrons to tunnel from the SL's into the QW, as compared to holes, due to the difference in effective mass. Consequently, the electron concentration in the QW can be varied accurately by the intensity of Ar-ion laser illumination with a radiation energy $\left(\hbar \omega_{\mathrm{ill}}=2.41 \mathrm{eV}\right)$ exceeding the SL band gap. Photoluminescence (PL), PL excitation (PLE), and reflectivity spectra were measured at $1.6 \mathrm{~K}$ and in magnetic fields up to $20 \mathrm{~T}$ applied perpendicular to the QW layers. An acousto-optical modulator with a time resolution of $100 \mathrm{~ns}$ was used in time-resolved experiments. A dye laser with a photon energy $\left(\hbar \omega_{\mathrm{dir}}\right)$ below the SL band gap was used for direct creation of excitons in the QW.

The PL spectrum of the QW, which consists of two lines, is shown in Fig. 1. The $X$ line is due to recombination of excitons, and has a linewidth of $1.5 \mathrm{meV}$. The $X^{-}$line, corresponding to the negatively charged exciton, is at $3.5 \mathrm{meV}$ lower energy than the $X$ line [2]. Increasing the background electron concentration by illuminating leads to an enhanced intensity of the $X^{-}$line relative to the $X$ line, as expected for a charged exciton. The temporal dependence of the intensity increase shows a rise time of $1.5 \mu$ s (see inset of Fig. 1). It is determined by the electron collection into the QW from the SL's. The decay time of the effect of $2.7 \mu \mathrm{s}$ is due to the indirect recombination of the QW electrons with holes located in the SL's. Note that the recombination time of excitons in the QW is 200 ps [7], which is much shorter than the discussed dynamical processes. The critical concentration for exciton screening in CdTe QW's equals units of $10^{11} \mathrm{~cm}^{-2}[2,8]$. We have not noticed exciton screening appearances in the studied structure up to illumination densities of $20 \mathrm{~W} / \mathrm{cm}^{2}$, where the illumination effect saturates. Therefore, the maximal electron concentration in the QW provided by the external illumination is estimated to be about $5 \times 10^{10} \mathrm{~cm}^{-2}$.

In an external magnetic field, a new line (ExCR line) appears in the PLE and reflectivity spectra which cannot be attributed to the normal magnetoexciton peaks [see 


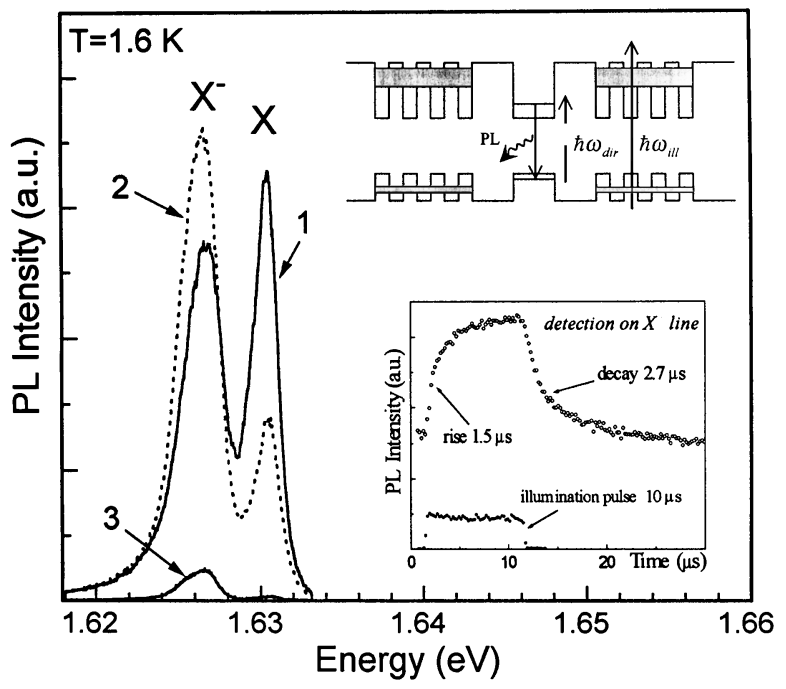

FIG. 1. Photoluminescence spectra of a $75 \AA \mathrm{CdTe} /$ $\mathrm{Cd}_{0.74} \mathrm{Mg}_{0.26} \mathrm{Te} \mathrm{QW}$ taken under different excitation conditions: 1 - direct excitation in the QW only, 2-direct excitation and illumination above the SL band gap, and 3-illumination only. The inset shows temporal changes of the $X^{-}$line intensity measured under pulsed illumination (open circles).

Fig. 2(a)]. The energy position of this ExCR line is in the range of the Coulomb bound states, but it behaves very differently. For instance, the intensity of the ExCR line increases strongly for higher illumination intensities (i.e., for larger $n_{e}$ ), whereas the magnetoexciton lines are insensitive to this factor [see Fig. 2(b)]. Furthermore, Fig. 3 shows that the ExCR line shifts linearly with magnetic field with a slope of $1.14 \mathrm{meV} / \mathrm{T}$, which is comparable to the electron cyclotron energy in $\mathrm{CdTe} /(\mathrm{Cd}, \mathrm{Mg}) \mathrm{Te} \mathrm{QW}$ 's. An extrapolation of this shift to zero field meets approximately
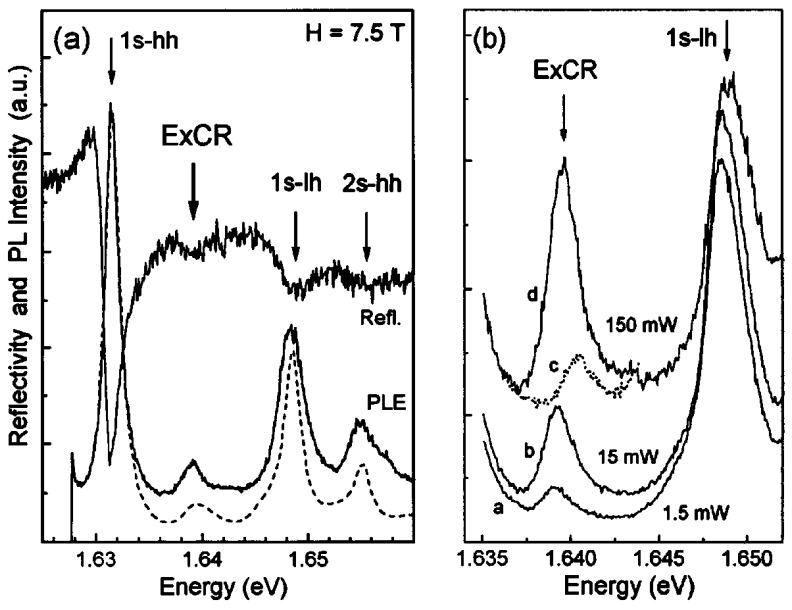

FIG. 2. (a) PLE and reflectivity spectra measure at a magnetic field of $7.5 \mathrm{~T}$. The signal was detected on the $X^{-}$line in $\sigma^{-}$circular polarization. The calculated absorption spectrum is shown by the dashed line. (b) PLE spectra taken under different intensities of the illumination (labeled in figure). Here, the PL is detected on the $X$ line in $\sigma^{-}$polarization, with $\sigma^{-}$and $\sigma^{+}$polarized excitation for the solid and dotted lines, respectively. the energy of the $1 s$ state of the heavy-hole exciton $(1 s$ $h h)$. This linear shift of the ExCR line, as opposed to the quadratic one for heavy-hole excitons $(1 s, 2 s, 3 s$, and $4 s$ states [10], also displayed in Fig. 3), shows that the free electrons contribute to the observed process. The Zeeman splitting of the ExCR line is similar to that of the $1 s-h h$ excitonic state. The ExCR line is strongly $\sigma^{-}$polarized [compare curves $c$ and $d$ in Fig. 2(b)], when the spin of the photoexcited electron is parallel to the free electron gas polarization in the external field direction [11].

The experimental behavior of the ExCR line indicates the following process: An incident photon creates an exciton and simultaneously excites a background electron from the zeroth to the first Landau level. The mechanism of the exciton-electron interaction responsible for such a combined exciton-cyclotron resonance is discussed below with the aim to establish theoretically how the background electrons modify the excitonic absorption spectrum in a magnetic field.

Considering a QW with $N$ electrons, $N$ being equal to the electron 2D density $n_{e}$ multiplied by the QW area, the absorption spectrum was calculated as the imaginary part of the polarizability $\chi_{\alpha \beta}$, given by the following equation:

$$
\chi_{\alpha \beta}=\frac{1}{2} \sum_{\Psi} \frac{\left\langle\Phi_{0}\left|P_{\alpha}\right| \Psi\right\rangle\left\langle\Psi\left|P_{\beta}\right| \Phi_{0}\right\rangle}{E_{\Psi}-E_{0}-\hbar \omega-i \delta} .
$$

Here $\left|\Phi_{0}\right\rangle$ and $|\Psi\rangle$ are the wave functions and $E_{0}$ and $E_{\Psi}$ are the energies of the ground and excited states of the system. $\left\langle\Phi_{0}\left|P_{\alpha}\right| \Psi\right\rangle$ is the matrix element of the $\alpha$ th component of the dipole moment operator $(\alpha=x, y, z)$, $\omega$ is the photon frequency, and $\delta \rightarrow 0$ is an infinitesimal imaginary increment of the frequency $\omega$.

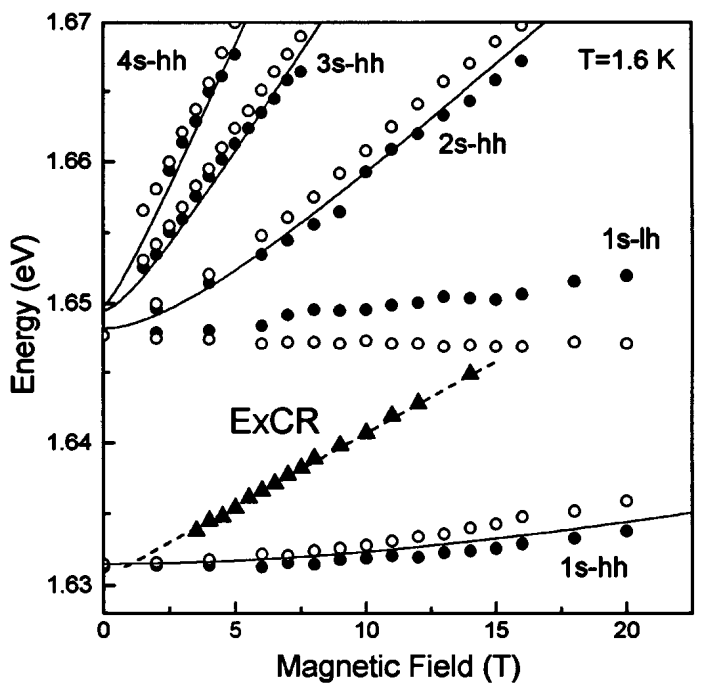

FIG. 3. Magnetoexciton fan chart of a $75 \AA$ CdTe/ $\mathrm{Cd}_{0.74} \mathrm{Mg}_{0.26} \mathrm{Te} \mathrm{QW}$. Closed and open symbols correspond to different spin orientations of the exciton states excited by $\sigma^{-}$ and $\sigma^{+}$polarized light, respectively. The solid lines show the result of a calculation according to Ref. [9]. The new ExCR line is plotted by triangles with a dashed line as a guide for the eye (only the strong $\sigma^{-}$polarized component is shown). 
The following approximations were used:

(i) The carrier cyclotron energies $\hbar \omega_{c, e(h)}=$ $e \hbar H / m_{e(h) c}$ are much smaller than the energy distance between quantum confined levels and also much smaller than the exciton binding energy, i.e., the exciton Bohr radius $a_{B} \ll L_{H}$, where $L_{H}=\sqrt{\hbar c / e H}$ is the magnetic length. In this case, modification of the exciton wave function by a magnetic field can be neglected.

(ii) The exciton states were treated as purely two dimensional.

(iii) All background electrons occupy the lowest Landau level with their spins oriented parallel to the field direction: $\sigma=1,(\uparrow) ; k T \ll \hbar \omega_{c, e}$ and $k T \ll \mu_{B} g_{e} H$, where $\mu_{B}$ is the Bohr magneton, $g_{e}$ is the electron $g$ factor, and $\sigma= \pm 1$ is the eigenvalue of the Pauli operator (low temperature limit). It is valid for the filling factor $f_{0}<1$. Because of inequality $a_{B} \ll L_{H}$, we have a condition for the electron concentration $n_{e} \ll 1 / a_{B}^{2}$. It means that electrons do not modify the excitons.

In what follows, the Zeeman splitting term will be omitted in order to simplify our equations. The spindependent properties will be discussed later in relation to the polarization properties of the ExCR line.

The ground state of the system is given by $\left|\Phi_{0}\right\rangle=$ $\prod_{X} a_{n=0, X}^{+}|0\rangle$, where $a_{n, X}^{+}$is the creation operator of a $2 \mathrm{D}$ electron in the $n$th Landau level with a Landau oscillator coordinate $X=L_{H}^{2} p_{y} / \hbar$, and $E_{0}=N \hbar \omega_{c, e} / 2$ is the ground state energy.

The excitonic states of the system are

(i) single exciton states: $\left|\Psi^{\mathbf{Q}}\right\rangle=C \iint d^{2} \mathbf{r}_{e} d^{2} \times$ $\mathbf{r}_{h} e^{i \mathbf{Q} \cdot \mathbf{R}} \phi\left(\mathbf{r}_{e}-\mathbf{r}_{h}\right) a^{+}\left(\mathbf{r}_{e}\right) b^{+}\left(\mathbf{r}_{h}\right)\left|\Phi_{0}\right\rangle$, where $a^{+}\left(\mathbf{r}_{e}\right)$ $\left[a\left(\mathbf{r}_{e}\right)\right]$ and $b^{+}\left(\mathbf{r}_{h}\right)$ are creation [annihilation] operators of an electron with a coordinate vector $\mathbf{r}_{e}$ and a heavy hole with coordinate vector $\mathbf{r}_{h} . \quad \mathbf{R}$ is an in-plane coordinate and $\mathbf{Q}$ is the wave vector of the exciton center of mass, and $\phi\left(\mathbf{r}_{e}-\mathbf{r}_{h}\right)$ is the exciton envelope wave function. The energy of the state $\left|\Psi^{\mathbf{Q}}\right\rangle$ measured from $E_{0}$ is $E_{\mathbf{Q}}=E_{\text {ex }}(\mathbf{Q})$ and at $\mathbf{Q}=0$ it corresponds to the exciton resonance.

(ii) excited states, responsible for the combined ExCR, with one exciton with momentum $\mathbf{Q}$ and one electron excited from Landau level $n=0$ into Landau level $n>$ $0: \quad\left|\Psi_{n, X ; 0, X_{\varphi}}^{\mathbf{Q}}\right\rangle=C^{\prime} \iint d^{2} \mathbf{r}_{e} d^{2} \mathbf{r}_{h} e^{i \mathbf{Q} \cdot \mathbf{R}} \phi\left(\mathbf{r}_{e}-\mathbf{r}_{h}\right) a^{+} \times$ $\left(\mathbf{r}_{e}\right) b^{+}\left(\mathbf{r}_{h}\right) a_{n, X}^{+} a_{0, X_{0}}\left|\Phi_{0}\right\rangle$. The energy of this state is $E_{n, X ; 0, X_{0}}^{\mathbf{Q}}=E_{\mathrm{ex}}(\mathbf{Q})+n \hbar \omega_{c, e}$.

To calculate the dipole moment operator, the wave functions of the photocreated electron $[n(\geq 1), X]$ were expanded in the eigenfunctions of an electron in a magnetic field. In the ExCR process, the photoexcited hole $[n(\geq 1),-X]$ interacts with the background electron $\left(n=0, X_{0}\right)$ and forms an exciton with a momentum $\mathbf{Q}$. Therefore, the final state of this process is an exciton with momentum $\mathbf{Q}$ and an electron in the state $[n(=1), X]$. The probability for the exciton to receive momentum $\mathbf{Q}$ is proportional to the modulus squared of the $Q$ th Fourier component of the electron $\left(n=0, X_{0}\right)$ and hole $[n(\geq$ $1),-X]$ wave functions localized by the magnetic field on the characteristic length $L_{H}$. Calculation of this probability gives a value of $(n !)^{-1}\left(Q^{2} L_{H}^{2} / 2\right)^{n} \exp \left(-Q^{2} L_{H}^{2} / 2\right)$, which appears in Eq. (2) in the ExCR term. The maximum of this probability corresponds to $Q=\sqrt{2 n} / L_{H}$. We emphasize that this theoretical approach accounts for all orders of perturbation theory.

The optical spectrum is given (in lowest order of the parameter $n_{e} a_{B}^{2}$ ) by

$$
\begin{aligned}
\operatorname{Im} \chi_{\alpha \beta}= & \pi d^{2}|\phi(0)|^{2} \delta\left(\hbar \omega-E_{\mathrm{ex}}(0)\right) \delta_{\alpha \beta} \\
& +n_{e} d^{2}\left|\int d^{2} \mathbf{r} \phi(\mathbf{r})\right|^{2} \int \frac{d^{2} \mathbf{Q}}{4 \pi} \exp \left(-Q^{2} L_{H}^{2} / 2\right) \sum_{n=1}^{\infty} \frac{1}{n !}\left(\frac{Q^{2} L_{H}^{2}}{2}\right)^{n} \delta\left[\hbar \omega-E_{\mathrm{ex}}(\mathbf{Q})-n \hbar \omega_{c, e}\right] \delta_{\alpha \beta} .
\end{aligned}
$$

Here, $d$ is the band-to-band dipole matrix element. The first term of Eq. (2) reflects the free exciton transition and the second term corresponds to the combined ExCR state. For a quadratic exciton dispersion $E_{\mathrm{ex}}(\mathbf{Q})=E_{\mathrm{ex}}(0)+$ $\hbar^{2} Q^{2} / 2 M$, with $M=m_{e}+m_{h}$, simply integrating the ExCR term gives

$$
\begin{aligned}
\operatorname{Im} \chi_{\alpha \beta}^{\mathrm{ExCR}}= & 4 \pi \delta_{\alpha \beta} n_{e} a_{B}^{2} d^{2} \frac{M}{\hbar^{2}} \sum_{n=1}^{\infty} \frac{1}{n !}\left(\frac{M}{m_{e}} \frac{\Delta \Omega_{n}}{\hbar \omega_{c, e}}\right)^{n} \\
& \times \exp \left(-\frac{M}{m_{e}} \frac{\Delta \Omega_{n}}{\hbar \omega_{c, e}}\right),
\end{aligned}
$$

where $\Delta \Omega_{n}=\hbar \omega-E_{\mathrm{ex}}(0)-n \hbar \omega_{c, e}$. It follows from Eqs. (2) and (3) that the absorption spectrum of the system in magnetic fields consists of a free exciton line and several lines shifted to higher energies. The peak positions of these additional lines correspond to energy distances of $n \hbar \omega_{c, e}\left(1+m_{e} / M\right), n=1,2,3, \ldots$, with respect to the exciton resonance. An estimation of the energy shift of the $n=1$ state with the masses $m_{e}=$ $0.11 m_{0}$ and $m_{h}=0.48 m_{0}$ [10] gives $1.24 \mathrm{meV} / \mathrm{T}$, close to the measured shift of the ExCR line of $1.14 \mathrm{meV} / \mathrm{T}$.

So far, we have neglected, in the ExCR term, the Coulomb interaction between the electron and the hole created by the photon. This interaction manifests itself in the formation of an exciton that plays the role of a virtual state. Including this interaction results in a strong increase of the ExCR contribution to the polarizability by a resonant factor $\left[4 E_{\mathrm{ex}}(0) / \omega_{c, e}\right]^{2} \approx 2 L_{H}^{4} / a_{B}^{4}$, by which the right part of Eq. (3) and the second term in Eq. (2) should be multiplied. The calculated ratio of the integral intensity of the first combined peak to the intensity of the exciton line $S_{0}$ is $S^{\mathrm{ExCR}} / S_{0}=8 n_{e} L_{H}^{2}=4 f_{0} / \pi$, where $f_{0}$ is the electron occupancy of the zero Landau level. We calculate the excitonic absorption spectrum at $7.5 \mathrm{~T}$, taking into account the resonant factor and using $f_{0}$ as a fitting parameter [see the dashed line in Fig. 2(a)]. The 


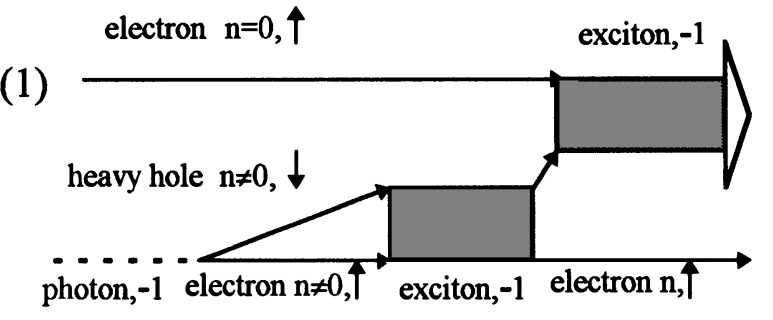

(2)

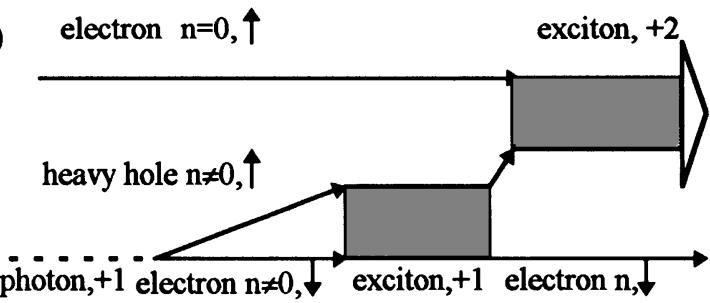

FIG. 4. Diagrams of the combined exciton-cyclotron resonance without (1) and with (2) change of the electron spin orientation between the initial and final states.

best agreement with the experimental PLE trace at $7.5 \mathrm{~T}$ was obtained by using a filling factor of $f_{0}=0.1$, which corresponds to $n_{e}=1.8 \times 10^{10} \mathrm{~cm}^{-2}$.

The strong circular polarization of the ExCR line in the PLE spectrum [see Fig. 2(b)] can also be explained by the model, which is illustrated by the diagrams in Fig. 4. Diagram 1 corresponds to $\sigma^{-}$polarization of the photon, which creates an electron with the same spin orientation as the background electron. In this case, the exciton angular momentum in the final state is equal to -1 , and the recombination of such an exciton is dipole allowed. The $\sigma^{+}$polarized photon leads to a final state exciton with a magnetic moment of 2 , of which the recombination is dipole forbidden (see diagram 2). Such excitons can recombine only after a spin flip of either the electron or the hole caused by scattering processes, and therefore their contribution to the PL intensity is much weaker.

We suppose the exciton Bohr radius to be much smaller than the magnetic length. This approximation is valid for magnetic fields less than 10-15 T. At higher fields, the squeezing of the exciton wave function will result in a decrease of the ExCR amplitude. In the present approach, we neglected the corrections to the eigenvalues and eigenfunctions originating from the Coulomb interaction between the electrons in the QW. We suppose that no Wigner crystallization phase transition occurs in our electron system, since the Coulomb electron-electron interaction is smaller than the temperature or electron level inhomogeneous broadening due to potential fluctuations in the QW. Under this condition, the corrections can be neglected because they give a small, of the order of $n_{e} a_{B}^{2}$, shift of the ExCR frequency and a small, of the order of $\left(n_{e} a_{B}^{2}\right)^{2}$, correction to the ExCR amplitude.

An alternative explanation, namely, that the ExCR originates from the interaction of the photogenerated exciton with background electrons due to exciton polarization (induced by the electrons), can be ruled out since the amplitude of this process is proportional to $\varepsilon^{-2}(\varepsilon \approx 10$ is the dielectric constant). Therefore, this process gives a small contribution in absorption (proportional to $\varepsilon^{-4}$ ) that does not depend on polarization.

In summary, the excitonic energy states of a $\mathrm{QW}$ containing a 2DEG of low density in the presence of a magnetic field has been found to possess new states, namely, a combined exciton-cyclotron resonance, which has indeed been observed experimentally in the PLE and reflectivity spectra. The theory shows that, in contrast to the conventional excitonic absorption where the exciton is formed from the photocreated electron and hole, the dominating mechanism responsible for this exciton-cyclotron resonance is the formation of an exciton from the photocreated hole and a background electron.

The authors acknowledge useful discussions with V.I. Perel, I. A. Merkulov, E. L. Ivchenko, R. T. Cox, and I. Bar-Joseph. This work has been supported by the European Commission (Contract No. CHGT-CT93-0051), Deutsche Forschungsgemeinschaft (SFB 410), and Russian Foundation for Basic Research (Grants No. 95-0204061a and No. 96-02-17952).

[1] J. C. Maan, in The Physics of Low-Dimensional Semiconductor Structures, edited by P. N. Butcher et al. (Plenum Press, New York, 1993), p. 333.

[2] K. Kheng et al., Phys. Rev. Lett. 71, 1752 (1993).

[3] G. Finkelstein, H. Shtrikman, and I. Bar-Joseph, Phys. Rev. Lett. 74, 976 (1995); H. Buhmann et al., Phys. Rev. B 51, 7969 (1995); A. J. Shields et al., Phys. Rev. B 51, 18049 (1995).

[4] M. Koch et al., Phys. Rev. B 51, 13887 (1995).

[5] B. M. Ashkinadze et al., Phys. Rev. B 51, 1938 (1995).

[6] R. Harel et al., Phys. Rev. B 53, 7868 (1996).

[7] W. Ossau et al., Superlattices Microstruct. 16, 5 (1994).

[8] V.D. Kulakovskii et al., Phys. Rev. B 54, 4981 (1996).

[9] A. H. MacDonald and D. S. Ritchie, Phys. Rev. B 33, 8336 (1986).

[10] The pattern of heavy-hole exciton states in magnetic field is fitted by the procedure for the two-dimensional magnetoexcitons suggested in Ref. [9]. A reduced exciton mass of $0.085 m_{0}$ has been determined as fitting parameter. It leads to electron and in-plane heavy-hole effective masses: $m_{e}=0.11 m_{0}$ and $m_{h}=0.48 m_{0}$.

[11] In CdTe $g_{e}=-1.77$ and at the temperature of $1.6 \mathrm{~K}$ $\mu_{B} g_{e} H>k T$ is satisfied for magnetic fields stronger than $1.5 \mathrm{~T}$. 